\title{
UNSTEADY LOW REYNOLDS NUMBER FLOW IN A HEATED TUBE OF SLOWLY VARYING SECTION
}

\author{
A. R. BESTMAN ${ }^{1,2}$ \\ (Received 31 January 1984; revised 1 August 1986)
}

\begin{abstract}
Fluid motion established by an oscillatory pressure gradient superimposed on a mean, in a tube of slowly varying section, is studied when the temperature of the tube wall varies with axial distance. Particular attention is focussed on the mean flow and steady streaming components of the oscillatory flow of higher approximation. For the velocity components, the axial component takes the pride of place, since this component is responsible for convection of nutrients to various parts of the body of a mammal in systematic circulation. A salient point in the paper concerns consequences of free convection currents at a constriction (stenosis).
\end{abstract}

\section{Introduction}

In a previous analysis, Bestman [1] studied the steady flow in a uniformly heated tube of slowly varying section in the presence of a heat source/sink term, as applicable to physiological situations. The temperature of the body, that is the temperature in the interior, called the core temperature, is fairly well regulated, normally varying from the mean by not more than $0.6^{\circ} \mathrm{C}$. Hence the wall of the blood vessels which travel deep inside the body will not show much variation in temperature, and the above analysis is applicable in such cases.

On the other hand, the surface temperature of the body rises and falls with the temperature of the surroundings, within a certain limit. In such a case, the wall temperature of blood vessels which start from the core to the surface

\footnotetext{
${ }^{1}$ Mathematics Programme, The Federal University of Technology, Yelwa Campus, P.M.B. 0248, Bauchi, Nigeria.

${ }^{2}$ Present address: Department of Mathematics, Statistics and Computer Science, University of Port Harcourt, Choba, P. M. B. 5323, Port Harcourt, Nigeria.

(C) Copyright Australian Mathematical Society 1988, Serial-fee code 0334-2700/88
} 
will exhibit variation with axial distance. An example is the subclavian artery which branches from the aorta and bifurcates further into the brachial artery that subsequently travels to the near surface of the body. The present problem, which considers a pulsating flow in a tube of varying section with axial temperature variation, is therefore addressed to these classes of blood vessels.

Also, a heat source/sink term is incorporated in the analysis to accommodate abnormalities of body temperature regulation in a pathological situation. For example, the thermal regulation centre in the hypothelamus may adjust its setpoint temperature to higher values when there is fever. Some causes of fever are bacterial diseases with subsequent decrease of white blood cells, brain tumors and a cycle of heat production.

The subsequent analysis is divided into five sections.

In Section 2 the non-dimensional equations are presented, followed by a determination of the leading approximations. In Section 3 the higher approximate solutions due to the mean pressure gradient are deduced in the light of [1]. The steady streaming solutions which appear at the higher approximations for the oscillatory pressure gradient are discussed in Sections 4 and 5. In Section 6 the results are discussed and quantitative appraisal of the steady state streaming are presented for a locally constricted tube.

\section{Governing equations and leading approximations}

We consider viscous flow, with heat source/sink term $Q$ in a heated long tube, which in cylindrical polar coordinates $\left(r^{\prime}, \phi, z^{\prime}\right)$ is defined as

$$
r^{\prime}=a\left(z^{\prime}\right)=a_{0} s\left(\varepsilon z^{\prime} / a_{0}\right) \quad 0<\varepsilon<1 .
$$

Here $a_{0}$ is a characteristic radius. We assume that the pipe is subjected to an axial pressure gradient at one end and $z^{\prime}=0$. Thus

$$
-\frac{\partial p^{\prime}}{\partial z^{\prime}}\left(r^{\prime}, \phi, 0, t^{\prime}\right)=C_{0}^{\prime}+K_{0}^{\prime} \sin \omega t^{\prime}
$$

The boundary conditions for the problem are the no slip condition for velocity and the equality of fluid and wall temperatures at the tube wall. Thus

$$
u^{\prime}=0=v^{\prime}=w^{\prime}, \quad T=T_{w} T_{a}\left(1+\lambda^{\prime} f\left(z^{\prime}\right)\right) .
$$

We have defined $\left(u^{\prime}, v^{\prime}, w^{\prime}\right)$ as the velocity components in the $\left(r^{\prime}, \phi, z^{\prime}\right)$-direction, $t^{\prime}$ is time, $\omega$ is frequency of oscillation and $T$ is the fluid temperature. $C_{0}^{\prime}$ and $K_{0}^{\prime}$ are the amplitudes of the pulsating pressure gradient and $T_{a, w, \infty}$ represent the atmospheric, wall and ambient temperatures respectively. We shall take the ambient temperature as that of the static blood in the left ventricle. 
Next we assume that the Boussinesq approximation is valid, so that if $\rho$ designates fluid density, the equation of state of the Boussinesq fluid is

$$
\rho_{\infty}-\rho=\rho_{\infty} \beta\left(T-T_{\infty}\right)
$$

where $\beta$ is the coefficient of volume expansion. Furthermore, let $\mu$ designate the fluid viscosity, $k$ the thermal conductivity and $c_{p}$ the specific heat at constant pressure. It is now expedient to introduce

$$
\begin{gathered}
t=\omega t^{\prime}, \quad r=r^{\prime} / a_{0}, \quad z=\varepsilon z^{\prime} / a_{0}, \\
(u, v, w)=\left(u^{\prime}, v^{\prime}, \varepsilon w^{\prime}\right) / \varepsilon U_{0}, \quad p=\left(p^{\prime}-p_{\infty}\right) a_{0} / \mu U_{0} \\
\theta=\frac{T-T_{\infty}}{T_{0}-T_{\infty}}, \quad\left(C_{0}, K_{0}\right)=\left(C_{0}^{\prime}, K_{0}^{\prime}\right) a_{0}^{2} / \mu U_{0} \\
\lambda=\frac{\lambda^{\prime} T_{0}}{T_{a}-T_{\infty}}, \quad \alpha=\frac{Q a_{0}^{2}}{K\left(T_{a}-T_{\infty}\right)}, \\
R=\rho_{\infty} U_{0} a_{0} / \mu, \quad G=\rho_{\infty} g \beta a_{0}^{2}\left(T_{a}-T_{\infty}\right) / \varepsilon \mu U_{0} \\
P=\mu c_{p} / k, \quad \sigma=\rho_{\infty} \omega a_{0}^{2} / \mu .
\end{gathered}
$$

Here $U_{0}$ is a typical axial velocity, $R$ is the Reynolds number of the flow such that $\varepsilon R=O(1)$ (the low Reynolds number assumption), $G$ is the free convection parameter or Grashof number, $P$ is the Prandtl number and $\sigma^{1 / 2}$ is usually referred to as the Womersley parameter. The gravitation $g$ makes an angle $\chi$ with the radial axis of the tube.

By virtue of (2.4) and (2.5), the non-dimensional equations of continuity, momentum and energy could be written as

$$
\begin{gathered}
\frac{1}{r} \frac{\partial}{\partial r}(r u)+\frac{1}{r} \frac{\partial v}{\partial \phi}+\frac{\partial w}{\partial z}=0 \\
\varepsilon \sigma \frac{\partial u}{\partial t}+\varepsilon^{2} R\left(u \frac{\partial u}{\partial r}+\frac{v}{r} \frac{\partial u}{\partial \phi}-\frac{v^{2}}{r}+w \frac{\partial u}{\partial z}\right) \\
=-\frac{\partial p}{\partial r}+\varepsilon\left(\nabla^{2}-\frac{1}{r}\right) u-\frac{2 \varepsilon}{r^{2}} \frac{\partial v}{\partial \phi}+\varepsilon^{3} \frac{\partial^{2} u}{\partial z^{2}}-\varepsilon \theta G \cos \chi \cos \phi \\
\varepsilon \sigma \frac{\partial v}{\partial t}+\varepsilon^{2} R\left(u \frac{\partial v}{\partial r}+\frac{v}{r} \frac{\partial v}{\partial \phi}+\frac{u v}{r}+w \frac{\partial v}{\partial z}\right) \\
=-\frac{1}{r} \frac{\partial p}{\partial \phi}+\varepsilon\left(\nabla^{2}-\frac{1}{r}\right) v+\frac{2 \varepsilon}{r^{2}} \frac{\partial u}{\partial \phi}+\varepsilon^{3} \frac{\partial^{2} v}{\partial z^{2}}+\varepsilon \theta G \cos \chi \sin \phi, \\
\sigma \frac{\partial w}{\partial t}+\varepsilon R\left(u \frac{\partial w}{\partial r}+\frac{v}{r} \frac{\partial w}{\partial \phi}+w \frac{\partial w}{\partial z}\right)=-\varepsilon \frac{\partial p}{\partial z}+\nabla^{2} w+\varepsilon^{2} \frac{\partial^{2} w}{\partial z^{2}}-\varepsilon \theta G \sin \chi \\
P \sigma \frac{\partial \theta}{\partial t}+\varepsilon P R\left(u \frac{\partial \theta}{\partial r}+\frac{v}{r} \frac{\partial \theta}{\partial \phi}+w \frac{\partial \theta}{\partial z}\right)=\nabla^{2} \theta+\varepsilon^{2} \frac{\partial^{2} \theta}{\partial z^{2}}+\alpha, \quad(2.6 \mathrm{a}, \mathrm{b}, \mathrm{c}, \mathrm{d}, \mathrm{e})
\end{gathered}
$$


subject to the conditions

$-\frac{\partial p}{\partial z}(r, \phi, 0, t)=\frac{1}{\varepsilon}\left(C_{0}+K_{0} \sin t\right), \quad u=0=v=w, \theta=1+\lambda f(z) \quad$ on $r=s(z)$.

A supplementary equation which is found useful in analysis is obtained by eliminating the pressure gradients from equations $(2.6 \mathrm{~b}, \mathrm{c})$. That is

$$
\begin{gathered}
\varepsilon R\left\{\frac{1}{r} \frac{\partial}{\partial r}\left[r\left(u \frac{\partial v}{\partial r}+\frac{v}{r} \frac{\partial v}{\partial \phi}+\frac{u v}{r}+w \frac{\partial v}{\partial z}\right)\right]\right. \\
\left.-\frac{1}{r} \frac{\partial}{\partial \phi}\left(u \frac{\partial u}{\partial r}+\frac{v}{r} \frac{\partial u}{\partial \phi}-\frac{v^{2}}{r}+w \frac{\partial u}{\partial z}\right)\right\} \\
=\left(\nabla^{2}-\sigma \frac{\partial}{\partial t}\right)\left[\frac{1}{r} \frac{\partial}{\partial r}(r v)-\frac{1}{r} \frac{\partial u}{\partial \phi}\right]+G \cos \chi\left(\frac{\partial \theta}{\partial r} \sin \phi+\frac{1}{r} \frac{\partial \theta}{\partial \phi} \cos \phi\right) \\
+\frac{\varepsilon^{2}}{r^{2}} \frac{\partial^{2}}{\partial z^{2}}\left[\frac{\partial}{\partial r}(r v)-\frac{\partial u}{\partial \phi}\right]
\end{gathered}
$$

such that

$$
\nabla^{2}=\frac{\partial^{2}}{\partial r^{2}}+\frac{1}{r} \frac{\partial}{\partial r}+\frac{1}{r^{2}} \frac{\partial^{2}}{\partial \phi^{2}}
$$

Also we take

$$
\alpha=q_{1}+q_{2} \tau(t), \quad \tau(t)=\sin (\alpha t+\delta)
$$

The statement of the problem is now complete.

Since $\varepsilon$ is small, we seek asymptotic expansion for the velocity components and temperature of the form

$$
u=u_{0}+\varepsilon u_{1}+\ldots \text { etc. }
$$

while for the pressure we write

$$
p=(1 / \varepsilon) p_{0}+p_{1}+\ldots
$$

Substituting (2.10) and (2.11) into equations (2.6)-(2.9), we find that for the leading approximation

$$
\begin{gathered}
\frac{1}{r} \frac{\partial}{\partial r}\left(r u_{0}\right)+\frac{1}{r} \frac{\partial v_{0}}{\partial \phi}+\frac{\partial w_{0}}{\partial z}=0 \\
\frac{\partial p_{0}}{\partial r}=0=\frac{1}{r} \frac{\partial p_{0}}{\partial \phi} \\
\sigma \frac{\partial w_{0}}{\partial t}=\frac{\partial p_{0}}{\partial z}+V^{2} w_{0}, \quad P_{\sigma} \frac{\partial \theta_{0}}{\partial t}=\nabla^{2} \theta_{0}+q_{1}+q_{2} \tau(t) \\
\left(\nabla^{2}-\sigma \frac{\partial}{\partial t}\right)\left[\frac{1}{r} \frac{\partial}{\partial r}\left(r v_{0}\right)-\frac{1}{r} \frac{\partial u_{0}}{\partial \phi}\right]+G \cos \chi\left[\frac{\partial \theta_{0}}{\partial r} \sin \phi+\frac{1}{r} \frac{\partial \theta_{0}}{\partial \phi} \cos \phi\right]=0 \\
-\frac{\partial p_{0}}{\partial z}(r, \phi, 0, t)=\left(C_{0}+K_{0} \sin t\right), \quad u_{0}=0=v_{0}=w_{0}, \theta_{0}=1+\lambda f(z) \\
\text { on } r=s(z) .
\end{gathered}
$$


First of all we observe that $p_{0}=p_{0}(z)$ only. Now putting

$$
u_{0}=u_{0}^{(0)}(r, \phi, z)+u_{0}^{(1)}(r, \phi, z, t) \text { etc. }
$$

we have

$$
\begin{gathered}
\frac{1}{r} \frac{\partial}{\partial r}\left(r u_{0}^{(0)}\right)+\frac{1}{r} \frac{\partial v_{0}^{(0)}}{\partial \phi}+\frac{\partial w_{0}^{(0)}}{\partial z}=0 \\
\nabla^{2} w_{0}^{(0)}=\frac{d p_{0}^{(0)}}{d z} \equiv p_{0}^{(0) \prime}, \quad \nabla^{2} \theta_{0}^{(0)}=-q_{1} \\
\nabla^{2}\left[\frac{1}{r} \frac{\partial}{\partial r}\left(r v_{0}^{(0)}\right)-\frac{1}{r} \frac{\partial u_{0}^{(0)}}{\partial \phi}\right]+G \cos \chi\left[\frac{\partial \theta_{0}^{(0)}}{\partial r} \sin \phi+\frac{1}{r} \frac{\partial \theta_{0}^{(0)}}{\partial \phi} \cos \phi\right]=0 \\
-p_{0}^{(0) \prime}(0)=C_{0}, \quad u_{0}^{(0)}=0=v_{0}^{(0)}=w_{0}^{(0)}, \theta_{0}^{(0)}=1+\lambda f(z) \quad \text { on } r=s(z),
\end{gathered}
$$

where an accent or a subscript denotes differentiation with respect to $z$, and

$$
\begin{gathered}
\frac{1}{r} \frac{\partial}{\partial r}\left(r u_{0}^{(1)}\right)+\frac{1}{r} \frac{\partial v_{0}^{(1)}}{\partial \phi}+\frac{\partial w_{0}^{(1)}}{\partial z}=0 \\
\sigma \frac{\partial w_{0}^{(1)}}{\partial t}=-\frac{\partial p_{0}^{(1)}}{\partial z}+\nabla^{2} w_{0}^{(1)}, \quad P_{\sigma} \frac{\partial \theta_{0}^{(1)}}{\partial t}=\nabla^{2} \theta_{0}^{(1)}+q_{2} \sin (\alpha t+\delta) \\
\left(\nabla^{2}-\sigma \frac{\partial}{\partial t}\right)\left[\frac{1}{r} \frac{\partial}{\partial r}\left(r v_{0}^{(1)}\right)-\frac{1}{r} \frac{\partial u_{0}^{(1)}}{\partial \phi}\right] \\
+G \cos \chi\left[\frac{\partial \theta_{0}^{(1)}}{\partial \phi} \sin \phi+\frac{1}{r} \frac{\partial \theta_{0}^{(1)}}{\partial \phi} \cos \phi\right]=0
\end{gathered}
$$

$$
-\frac{\partial p_{0}^{(1)}}{\partial z}(0, t)=K_{0} \sin t, \quad u_{0}^{(1)}=0=v_{0}^{(1)}=w_{0}^{(1)}=\theta_{0}^{(1)} \quad \text { on } r=s(z) .
$$

The method of solution of (2.12) has been discussed by Bestman [1]. The results are

$$
\begin{gathered}
\theta_{0}^{(0)}=1+\lambda f(z)+\frac{1}{2} q_{1} s^{2}\left[1-(r / s)^{2}\right], \quad w_{0}^{(0)}=\frac{1}{16} C_{0} \frac{s^{4}(0)}{s^{2}(z)}\left[1-(r / s)^{2}\right], \\
p_{0}^{(0) \prime}=-C_{0} \frac{s^{4}(0)}{s^{4}(z)} \\
u_{0}^{(0)}=\frac{1}{4} C_{0} s^{4}(0) \frac{s_{z}}{s^{2}}\left[r / s-(r / s)^{3}\right]-\frac{1}{384} G \cos \chi q_{1} s^{4}\left[1-(r / s)^{2}\right]^{2} \cos \phi \\
v_{0}^{(0)}=\frac{1}{384} G \cos \chi q_{1} s^{4}\left[1-(r / s)^{2}\right]\left[1-5(r / s)^{2}\right] \sin \phi .
\end{gathered}
$$

It is pertinent to note that the wall temperature variation does not affect the basic axial flow variables. 
For the oscillatory components, we put

$$
\begin{gathered}
w_{0}^{(1)}=\frac{1}{2}\left(h_{0} e^{2 t}+\tilde{h}_{0} e^{-i t}\right), \quad \theta_{0}^{(1)}=\frac{1}{2}\left(\gamma_{0} e^{i(\alpha t+\delta)}+\gamma_{0} e^{-i(\alpha t+\delta)}\right), \\
p_{0}^{(1)}=\frac{1}{2 i}\left(P_{0}^{(1)} e^{i t}+\widetilde{P}_{0}^{(1)} e^{-i t}\right) \\
u_{0}^{(1)}=\frac{1}{2}\left(f_{0} e^{i t}+\tilde{f}_{0} e^{-i t}\right)+\frac{1}{2}\left(f_{1} e^{i(\alpha t+\delta)}+\tilde{f}_{1} e^{-i(\alpha t+\delta)}\right), \\
v_{0}^{(1)}=\frac{1}{2}\left(g_{1} e^{i(\alpha t+\delta)}+\tilde{g}_{1} e^{-i(\alpha t+\delta)}\right)
\end{gathered}
$$

Thus

$$
\begin{gathered}
\frac{1}{r} \frac{\partial}{\partial r}\left(r f_{0}\right)^{\prime}+\frac{1}{r} \frac{\partial g_{0}}{\partial \phi}+\frac{\partial h_{0}}{\partial z}=0, \quad \frac{1}{r} \frac{\partial}{\partial r}\left(r f_{1}\right)+\frac{1}{r} \frac{\partial g_{1}}{\partial \phi}=0 \\
\left(\nabla^{2}-i \sigma\right) h_{0}=\frac{\partial P_{0}^{(1)}}{\partial z}, \quad\left(\nabla^{2}-i \sigma P \alpha\right) \gamma_{0}=i q_{2} \\
\left(\nabla^{2}-i \sigma \alpha\right)\left[\frac{1}{r} \frac{\partial}{\partial r}\left(r g_{1}\right)-\frac{1}{r} \frac{\partial f_{1}}{\partial \phi}\right]=-G \cos \chi\left[\frac{\partial \gamma_{0}}{\partial r} \sin \phi+\frac{1}{r} \frac{\partial \gamma_{0}}{\partial \phi} \cos \phi\right], \\
-\frac{\partial P_{0}^{(1)}}{\partial z}(0)=i K_{0}, \quad f_{0}=f_{1}=g_{1}=h_{0}=\gamma_{0}=0 \quad \text { on } r=s(z) .
\end{gathered}
$$

Then

$$
\begin{gathered}
h_{0}=-\frac{P_{0}^{(1) \prime}}{i \sigma}\left[1-\frac{J_{0}(\varsigma r)}{J_{0}(\zeta s)}\right], \\
\gamma_{0}=-\frac{q_{2}}{\sigma P r \alpha}\left[1-\frac{J_{0}\left(P^{1 / 2} \alpha^{1 / 2} \zeta r\right)}{J_{0}\left(P^{1 / 2} \alpha^{1 / 2} \varsigma s\right)}\right],
\end{gathered}
$$

where $\varsigma^{2}=-i \sigma$ and $J_{m}(x)$ is Bessel's function of the first kind of order $m$. Next, we set

$$
f_{0}=f_{0}^{(0)}(r, z), \quad f_{1}=f_{1}^{(0)}(r, z) \cos \phi, \quad g_{1}=g_{1}^{(0)}(r, z) \sin \phi
$$

with the result

$$
\frac{1}{r} \frac{\partial}{\partial r}\left(r f_{0}^{(0)}\right)=\frac{P_{0}^{(1) \prime \prime}}{i \sigma}\left[1-\frac{J_{0}(\varsigma r)}{J_{0}(\zeta s)}\right]-\frac{P_{0}^{(1) \prime}}{i \sigma} \zeta s^{\prime} \frac{J_{1}(\zeta s)}{J_{0}^{2}(\zeta s)} J_{0}(\varsigma r)
$$

and

$$
\begin{gathered}
\frac{1}{r} \frac{\partial}{\partial r}\left(r f_{1}^{(0)}\right)+\frac{1}{r} g_{1}^{(0)}=0 \\
\left(\frac{\partial^{2}}{\partial r^{2}}+\frac{1}{r} \frac{\partial}{\partial r}-\frac{1}{r^{2}}-i \sigma \alpha\right)\left[\frac{1}{r} \frac{\partial}{\partial r}\left(r g_{1}^{(0)}\right)+\frac{1}{r} f_{1}^{(0)}\right] \\
=G \cos \chi \cdot \frac{q_{2}}{\sigma \alpha^{1 / 2} P^{1 / 2}} \cdot \frac{J_{1}\left(P^{1 / 2} \alpha^{1 / 2} \zeta r\right)}{J_{0}\left(P^{1 / 2} \alpha^{1 / 2} \zeta s\right)}
\end{gathered}
$$


Integrating equation (19) gives

$$
f_{0}^{(0)}=\frac{P_{0}^{(1) \prime \prime}}{i \sigma}\left[\frac{1}{2} r-\frac{J_{1}(\varsigma r)}{\varsigma J_{0}(\varsigma s)}\right]-\frac{P^{(1) \prime}}{i \sigma} s^{\prime} \frac{J_{1}(\varsigma s)}{J_{0}^{2}(\varsigma s)} J_{1}(\varsigma r) .
$$

If we impose the no-slip condition on (2.21) we deduce the Reynolds equation for the pressure

$$
\left[\frac{1}{2} s^{2}-\frac{s J_{1}(\zeta s)}{\zeta J_{0}(\zeta s)}\right] P_{0}^{(1) \prime \prime}-s s^{\prime}\left[\frac{J_{1}(\varsigma s)}{J_{0}(\varsigma s)}\right]^{2} P_{0}^{(1) \prime}=0
$$

as first derived by Hall [3]. In virtue of the pressure boundary condition in (2.16), (2.22) reduces to

$$
P_{0}^{(1) \prime}=i K_{0}\left[\frac{\frac{1}{2} s^{2}(0)-s(0) J_{1}(\varsigma s(0)) / \varsigma J_{0}(\zeta s(0))}{\frac{1}{2} s^{2}-s J_{1}(\varsigma s) / \varsigma J_{0}(\zeta s)}\right] .
$$

Finally, integrating equation $(2.20 \mathrm{~b})$ once, we have

$$
\frac{1}{r} \frac{\partial}{\partial r}\left(r g_{1}^{(0)}\right)+\frac{1}{r} f_{1}^{(0)}=\frac{G \cos \chi q_{2}}{\sigma \alpha P^{1 / 2}(1-P)}\left[B_{1}^{(0)} \cdot \frac{1}{P} J_{1}\left(\alpha^{1 / 2} \zeta r\right)+J_{1}\left(P^{1 / 2} \alpha^{1 / 2}{ }^{\prime} r\right)\right],
$$

and then solving this equation simultaneously with (2.20a), we can show that

$$
\begin{aligned}
f_{1}^{(0)}= & \frac{i G \cos \chi q_{2}}{\sigma^{2} \alpha^{2} P^{3 / 2}(1-P)}\left[A_{1}^{(0)}+B_{1}^{(0)} \frac{J_{1}\left(\alpha^{1 / 2} \varsigma r\right)}{r}+\frac{J_{1}\left(P^{1 / 2} \alpha^{1 / 2} \varsigma r\right)}{r}\right] \\
g_{1}^{(0)}= & \frac{-i G \cos \chi q_{2}}{\sigma^{2} \alpha^{2} P^{3 / 2}(1-P)}\left\{A_{1}^{(0)}+B_{1}^{(0)} \alpha^{1 / 2} \varsigma\left[J_{0}\left(\alpha^{1 / 2} \varsigma r\right)-\frac{1}{\alpha^{1 / 2} \varsigma r} J_{1}\left(\alpha^{1 / 2} \varsigma r\right)\right]\right. \\
& \left.\quad+P^{1 / 2} \alpha^{1 / 2} \varsigma\left[J_{0}\left(P^{1 / 2} \alpha^{1 / 2} \varsigma r\right)-\frac{1}{P^{1 / 2} \alpha^{1 / 2} \varsigma r} J_{1}\left(P^{1 / 2} \alpha^{1 / 2} \zeta r\right)\right]\right\}
\end{aligned}
$$

where $A_{1}^{(0)}$ and $B_{1}^{(0)}$ are arbitrary functions of $z$ given by

$$
\begin{aligned}
A_{1}^{(0)} & =\frac{\alpha^{1 / 2} \zeta\left[P^{1 / 2} J_{0}\left(P^{1 / 2} \alpha^{1 / 2} \zeta s\right) J_{1}\left(\alpha^{1 / 2} \zeta s\right)-J_{0}\left(\alpha^{1 / 2} \zeta s\right) J_{1}\left(P^{1 / 2} \alpha^{1 / 2} \zeta s\right)\right]}{\alpha^{1 / 2} \zeta s J_{0}\left(\alpha^{1 / 2} \zeta s\right)-2 J_{1}\left(\alpha^{1 / 2} \zeta s\right)} \\
B_{1}^{(0)} & =\frac{2 J_{1}\left(P^{1 / 2} \alpha^{1 / 2} \zeta s\right)-P^{1 / 2} \alpha^{1 / 2} \zeta s J_{0}\left(P^{1 / 2} \alpha^{1 / 2} \zeta s\right)}{\alpha^{1 / 2} \zeta s J_{0}\left(\alpha^{1 / 2} \zeta s\right)-2 J_{1}\left(\alpha^{1 / 2} \zeta s\right)}
\end{aligned}
$$

and the solution to the leading approximation is now complete.

We observe that the leading pressure gradient given by (2.23) differs from the forcing pressure in (2.16). In the absence of free convection currents, the two pressure gradients are the same (Bestman [2]). 


\section{Higher approximation for the mean pressure gradient}

If we continue the expansion started in (2.10) and (2.11), then the order $O(\varepsilon)$ approximation is

$$
\begin{gathered}
\frac{1}{r} \frac{\partial}{\partial r}\left(r u_{1}\right)+\frac{1}{r} \frac{\partial v_{1}}{\partial \phi}+\frac{\partial w_{1}}{\partial z}=0 \\
\frac{\partial p_{1}}{\partial r}=0=\frac{1}{r} \frac{\partial p_{1}}{\partial \phi} \\
\sigma \frac{\partial w_{1}}{\partial t}+R\left(u_{0} \frac{\partial w_{0}}{\partial \phi}+\frac{v_{0}}{r} \frac{\partial w_{0}}{\partial \phi}+w_{0} \frac{\partial w_{0}}{\partial z}\right)=-\frac{\partial p_{1}}{\partial z}+\nabla^{2} w_{1}-G \sin \chi \theta_{0} \\
P \sigma \frac{\partial \theta_{1}}{\partial t}+P R\left(u_{0} \frac{\partial \theta_{0}}{\partial r}+\frac{v_{0}}{r} \frac{\partial \theta_{0}}{\partial \phi}+w_{0} \frac{\partial \theta_{0}}{\partial z}\right)=\nabla^{2} \theta_{1} \\
\left(\nabla^{2}-\sigma \frac{\partial}{\partial t}\right)\left[\frac{1}{r} \frac{\partial}{\partial r}\left(r v_{1}\right)-\frac{1}{r} \frac{\partial u_{1}}{\partial \phi}\right]+G \cos \chi\left(\frac{\partial \theta_{1}}{\partial r} \sin \phi+\frac{1}{r} \frac{\partial \theta_{1}}{\partial \phi} \cos \phi\right) \\
=R\left\{\frac{1}{r} \frac{\partial}{\partial r}\left[r\left(u_{0} \frac{\partial v_{0}}{\partial r}+\frac{v_{0}}{r} \frac{\partial v_{0}}{\partial \phi}+\frac{u_{0} v_{0}}{r}+w_{0} \frac{\partial v_{0}}{\partial z}\right)\right]\right. \\
\left.-\frac{1}{r} \frac{\partial}{\partial \phi}\left(u_{0} \frac{\partial u_{0}}{\partial r}+\frac{v_{0}}{r} \frac{\partial u_{0}}{\partial \phi}-\frac{v_{0}^{2}}{r}+w_{0} \frac{\partial u_{0}}{\partial z}\right)\right\},
\end{gathered}
$$

subject to the conditions

$$
\begin{gathered}
u_{1}=0=v_{1}=w_{1}=\theta_{1} \text { on } r=s(z), \\
\partial p_{1} / \partial z(r, \phi, 0, t)=0 .
\end{gathered}
$$

Once more we observe that $p_{1}=p_{1}(z, t)$ only. Again we seek solution in the form

$$
u_{1}=u_{1}^{(0)}(r, \phi, z)=u_{1}^{(1)}(r, \phi, z, t) \text { etc. }
$$

For the present case of discourse, $\sigma=0, K_{0}=0=q_{2}$ in (3.1) and (3.2) and by virtue of solution (2.14), (3.1c, d) can be integrated in a straightforward manner. The results are

$$
\begin{aligned}
\theta_{1}^{(0)}= & -\frac{1}{2.16^{2}} P R q_{2} C_{0} s^{4}(0) s s_{z}\left[11-18(r / s)^{2}+9(r / s)^{4}-2(r / s)^{6}\right] \\
& -\frac{1}{4.96^{2}} G \cos \chi P R q^{2} s^{7}\left[3(r / s)-6(r / s)^{3}+4(r / s)^{5}-(r / s)^{7}\right] \cos \phi \\
& +\frac{1}{25200} P G \cos \chi q_{1} \lambda s^{5} f_{z}\left[16(r / s)-35(r / s)^{3}+35(r / s)^{5}-16(r / s)^{7}\right] \cos \phi \\
& -\frac{3}{80} P \lambda C_{0} s^{4}(0) f_{z}\left[4(r / s)-5(r / s)^{2}+(r / s)^{4}\right] \cos \phi
\end{aligned}
$$


and

$$
\begin{aligned}
w_{1}^{(0)}= & -p_{1}^{(0) \prime} s^{2}\left[1-(r / s)^{2}\right] \\
& -\frac{1}{64^{2}} R C_{0}^{2} s^{8}(0) s^{2}\left(1 / s^{4}\right)_{z}\left[\frac{11}{9}-2(r / s)^{2}+(r / s)^{4}-\frac{2}{9}(r / s)^{6}\right] \\
& -\frac{1}{384^{2}} G \cos \chi R q_{1} C_{0} s^{4}(0) s^{3}\left[3(r / s)-6(r / s)^{3}+4(r / s)^{5}-(r / s)^{7}\right] \cos \phi \\
& +\frac{1}{25200} P G \cos \chi \lambda s^{2} f_{z}\left[16(r / s)-35(r / s)^{3}+35(r / s)^{5}-16(r / s)^{7}\right] \cos \phi \\
& -G \sin \chi\left\{[1+\lambda f(z)] s^{2}\left[1-(r / s)^{2}\right]+\frac{1}{8} q_{1} s^{3}\left[\frac{3}{4}-(r / s)^{2}+\frac{1}{4}(r / s)^{4}\right]\right\}
\end{aligned}
$$

The function $p_{1}^{(0) \prime}$ can be obtained by lubrication theory as discussed by Bestman [1]. In short to deduce the solution for $u_{0}^{(1)}$ and $v_{0}^{(1)}$, we write

$$
\begin{aligned}
u_{1}^{(0)}= & \frac{1}{64^{2}} C_{0}^{2} s^{8}(0) \hat{f}_{1}^{(0)}(r, z)+\frac{1}{64} C_{0} s^{4}(0) f_{1}^{(1)}(r, z) \cos \phi+F_{1}^{(1)}(r, z) \cos \phi \\
& +F_{2}^{(1)}(r, z) \cos 2 \phi+F_{2}^{(1)}(r, z) \cos 2 \phi, \\
v_{1}^{(0)}= & \frac{1}{64} C_{0} s^{4}(0) g_{1}^{(1)}(r, z) \sin \phi+G_{1}^{(1)}(r, z) \sin \phi+g_{2}^{(1)}(r, z) \sin 2 \phi \\
& +G_{2}^{(1)}(r, z) \sin 2 \phi .
\end{aligned}
$$

Then

$$
\begin{aligned}
p_{1}^{(0) \prime}= & \frac{1}{16} R C_{0} \frac{s^{4}(0)}{s^{4}}\left[\frac{s_{z}}{s}-\frac{s_{z}(0)}{s(0)}\right] \\
& -\frac{256^{2} G \sin \chi}{C_{0}^{2} s^{8}(0)} \cdot \frac{1}{s^{4}} \int_{s(0)}^{s}\left\{\frac{1}{4} \lambda s^{3} f_{z}+[1+\lambda f(z)] s^{2} s_{z}+\frac{10}{96} q_{1}^{2} s^{2} s_{z}\right\} d z
\end{aligned}
$$

and $\hat{f}_{1}^{(0)}, f_{1,2}^{(1)}, g_{1,2}^{(1)}$ are given in [1] by (3.22), (3.24), (3.25) respectively, except for an additional term in $\hat{f}_{1}^{(0)}$ which is a multiple of $G \sin \chi$. The remaining functions $F_{1,2}^{(1)}, G_{1,2}^{(1)}$, which depend on the axial temperature variation, may be obtained similarly. The additional term and these functions are given in the appendix.

\section{Steady state streaming for small $\sigma$}

Steady state streaming, which has attracted both mathematical and physiological interest, arises as a result of pure oscillatory forcing pressure, and is different from a steady solution that would be provoked by a time-independent 
pressure. The steady streaming that results from multiplication of exponentially time-varying functions manifests itself when $\alpha=1$ and $\delta=0$, and the results in this and the following sections are for this special case.

The cause of this steady-state streaming is the non-linearity of the governing equation. For example, if in the oscillatory components of (3.1) and (3.2) we employ a subscript $s$ to denote steady streaming components, we find that as before for the order $\theta(\varepsilon)$ approximation, the pressure $p_{s}^{(1)}=p_{s}^{(1)}(z)$. For the temperature and velocity components we write

$$
\begin{aligned}
\theta_{s}^{(1)} & =\gamma_{s}^{(0)}(r, z)+\gamma_{s}^{(1)}(r, s) \cos \phi \\
w_{s}^{(1)} & =h_{s}^{(0)}(r, z)+h_{s}^{(1)}(r, z) \cos \phi \\
u_{s}^{(1)} & =f_{s}^{(0)}(r, z)+f_{s}^{(1)}(r, z) \cos \phi+f_{s}^{(2)}(r, z) \cos 2 \phi \\
v_{s}^{(1)} & =g_{s}^{(1)}(r, z) \sin \phi+g_{s}^{(2)}(r, z) \sin 2 \phi .
\end{aligned}
$$

The remaining equations of this approximation become

$$
\begin{aligned}
& \frac{\partial^{2} \gamma_{s}^{(0)}}{\partial r^{2}}+\frac{1}{r} \frac{\partial \gamma_{s}^{(0)}}{\partial r}=\frac{1}{2} P R \cdot \operatorname{Re}\left[f_{0}^{(0)} \frac{\partial \tilde{\gamma}_{0}}{\partial r}+h_{0} \frac{\partial \tilde{\gamma}_{0}}{\partial z}\right] \\
& \frac{\partial^{2} \gamma_{s}^{(1)}}{\partial r^{2}}+\frac{1}{r} \frac{\partial \gamma_{s}^{(1)}}{\partial r}-\frac{1}{r^{2}} \gamma_{s}^{(1)}=\frac{1}{2} P R . \operatorname{Re}\left[f_{1}^{(0)} \frac{\partial \tilde{\gamma}}{\partial r}\right] \text {, } \\
& \frac{\partial^{2} h_{s}^{(0)}}{\partial r^{2}}+\frac{1}{r} \frac{\partial h_{s}^{(0)}}{\partial r}=\frac{1}{2} R \cdot \operatorname{Re}\left[f_{0}^{(0)} \frac{\partial \tilde{h}_{0}}{\partial r}+h_{0} \frac{\partial \tilde{h}_{0}}{\partial z}\right]+p_{s}^{\prime} \\
& \frac{\partial^{2} h_{s}^{(1)}}{\partial r^{2}}+\frac{1}{r} \frac{\partial h^{(1)}}{\partial r}-\frac{1}{r^{2}} h_{s}^{(1)}=\frac{1}{2} R \cdot \operatorname{Re}\left[f_{1}^{(0)} \frac{\partial \tilde{h}_{0}}{\partial r}\right] \\
& \frac{1}{r} \frac{\partial}{\partial r}\left(r f_{s}^{(0)}\right)=-\frac{\partial h_{s}^{(0)}}{\partial z} \\
& \frac{1}{r} \frac{\partial}{\partial r}\left(r f_{s}^{(1)}\right)+\frac{1}{r} g_{s}^{(1)}=-\frac{\partial h_{s}^{(1)}}{\partial z} \\
& \left(\frac{\partial^{2}}{\partial r^{2}}+\frac{1}{r} \frac{\partial}{\partial r}-\frac{1}{r^{2}}\right)\left[\frac{1}{r} \frac{\partial}{\partial r}\left(r g_{s}^{(1)}\right)+\frac{1}{r} f_{s}^{(1)}\right] \\
& =-\frac{1}{2} R \cdot \operatorname{Re}\left\{\frac{1}{r} \frac{\partial}{\partial r}\left[r\left(f_{0}^{(0)} \frac{\partial \tilde{g}_{1}^{(0)}}{\partial r}+\frac{1}{r} f_{0}^{(0)} \tilde{g}_{1}^{(0)}+w_{0}^{(0)} \frac{\partial \tilde{g}_{1}^{(0)}}{\partial z}\right)\right]\right. \\
& \left.+\frac{1}{r}\left(f_{0}^{(0)} \frac{\partial \tilde{f}_{1}^{(0)}}{\partial r}+f_{1}^{(0)} \frac{\partial \tilde{f}_{0}^{(0)}}{\partial r}\right)+\frac{1}{r} w_{0}^{(0)} \frac{\partial \tilde{f}_{1}^{(0)}}{\partial z}\right\} \\
& -G \cos \chi \frac{\partial \gamma_{s}^{(0)}}{\partial r}
\end{aligned}
$$


and

$$
\begin{gathered}
\frac{1}{r} \frac{\partial}{\partial r}\left(r f_{s}^{(2)}\right)+\frac{2}{r} g_{s}^{(2)}=0 \\
\left(\frac{\partial^{2}}{\partial r^{2}}+\frac{1}{r} \frac{\partial}{\partial r}-\frac{4}{r^{2}}\right)\left[\frac{1}{r} \frac{\partial}{\partial r}\left(r g_{s}^{(2)}\right)+\frac{2}{r} f_{s}^{(2)}\right] \\
=-\frac{1}{4} R \cdot \operatorname{Re}\left\{\frac{1}{r} \frac{\partial}{\partial r}\left[\left(f_{1}^{(0)} \frac{\partial \tilde{g}_{1}^{(0)}}{\partial r}+\frac{1}{r} g_{1}^{(0)} \tilde{g}_{1}^{(0)}+\frac{1}{r} f_{1}^{(0)} \tilde{g}_{1}^{(0)}\right)\right]\right. \\
\left.+\frac{2}{r^{2}}\left(\tilde{f}_{1}^{(0)} g_{1}^{(0)}+g_{1}^{(0)} \tilde{g}_{1}^{(0)}\right)+\frac{2}{r} f_{1}^{(0)} \frac{\partial \tilde{f}_{1}^{(0)}}{\partial r}\right\} \\
-G \cos \chi \cdot r \frac{\partial}{\partial r}\left(\frac{\gamma_{s}^{(1)}}{r}\right) .
\end{gathered}
$$

Here Re represents the real part of a complex variable. These quantities are to be solved subject to homogeneous boundary conditions at the wall $r=s(z)$ for the velocity components and the temperature and the pressure condition $p_{s}^{\prime}(0)=0$.

Because of the transcendental nature of the right-hand side of equations (4.1)(4.5), we shall discuss the solution to these equations for small and large values of $\sigma$ only. The oscillatory solutions could be tackled similarly.

When $\sigma$ is small, it is possible to obtain uniformly-valid approximate solutions to equations (4.1)-(4.5). For example, substituting (2.17) and (2.21) in (4.1) and (4.2) and taking the limit as $\sigma \rightarrow 0$, we have

$$
\begin{aligned}
& \frac{\partial^{2} \gamma_{s}^{(0)}}{\partial r^{2}}+\frac{1}{r} \frac{\partial \gamma_{s}^{(0)}}{\partial r}=-\frac{1}{16} \sigma \mathrm{PRK}_{0} q_{2} \frac{s^{4(0)}}{s^{4}} \cdot \frac{s^{\prime}}{s}\left(s^{2}-r^{2}\right)^{2}+O(\sigma) \\
& \frac{\partial^{2} \gamma_{s}^{(1)}}{\partial r^{2}}+\frac{1}{r} \frac{\partial \gamma_{s}^{(1)}}{\partial r}-\frac{1}{r^{2}} \gamma_{s}^{(1)}=-\frac{1}{1536} G \cos \chi q_{2}^{2}(\sigma / 2)^{1 / 2}\left[r^{5}-s^{4} r+O(\sigma)\right]
\end{aligned}
$$

and

$$
\begin{aligned}
& \frac{\partial^{2} h_{s}^{(0)}}{\partial r^{2}}+\frac{1}{r} \frac{\partial h_{s}^{(0)}}{\partial r}=-\frac{1}{16} R K_{0}^{2} \frac{s^{8}(0)}{s^{8}} \cdot \frac{s^{\prime}}{s}\left(s^{2}-r^{2}\right)\left(s^{2}-3 r^{2}\right)+p_{s}^{\prime}+O(\sigma) \\
& \frac{\partial^{2} h_{s}^{(1)}}{\partial r^{2}}+\frac{1}{r} \frac{\partial h_{s}^{(1)}}{\partial r}-\frac{1}{r^{2}} h_{s}^{(1)} \\
& =-\frac{1}{1536} K_{0} G \cos \chi q_{2} \cdot \frac{s^{4}(0)}{s^{4}}(\sigma / 2)^{1 / 2}\left[r^{5}-s^{4} r+O(\sigma)\right]
\end{aligned}
$$


The solutions of these equations subject to homogeneous conditions at the tube wall are

$$
\begin{aligned}
\gamma_{s}^{(0)}= & \frac{1}{1152} \alpha P R K_{0} q_{2} \frac{S^{4}(0)}{S^{4}} \cdot s^{5} s^{\prime}\left[11-18(r / s)^{2}+9(r / s)^{4}-2(r / s)^{6}\right]+O(\sigma), \\
\gamma_{s}^{(1)}= & \frac{1}{32.48} G \cos \chi q_{2}^{2} s^{7}(\sigma / 2)^{1 / 2}\left[5(r / s)-6(r / s)^{5}+(t / s)^{7}+O(\sigma)\right] \\
h_{s}^{(0)}= & -\frac{1}{4} p_{s}^{\prime} s^{2}\left[1-(r / s)^{2}\right] \\
& +\frac{1}{192} K_{0}^{2} R \frac{s^{8}(0)}{s^{8}} \cdot s^{5} s^{\prime}\left[1-3(r / s)^{2}+3(r / s)^{4}-(r / s)^{6}\right]+O(\sigma),
\end{aligned}
$$

and

$$
h_{s}^{(1)}=\frac{1}{32.48^{2}} K_{0} G \cos \chi q_{2} \cdot \frac{s^{4}(0)}{s^{4}} \cdot s^{7}(\sigma / 2)^{1 / 2}\left[5(r / s)-6(r / s)^{3}+(r / s)^{7}\right] .
$$

Substituting (4.8) in (4.3) and integrating we obtain

$$
\begin{aligned}
f_{s}^{(0)}= & \frac{1}{16} p_{s}^{\prime \prime} s^{3}\left[2(r / s)^{3}\right]+\frac{1}{4} p_{s}^{\prime} s^{2} s^{\prime}(r / s) \\
& -\frac{1}{1536} K_{0}^{2} R \frac{s^{8}(0)}{s^{8}} \cdot s^{6} s^{\prime \prime}\left[4(r / s)-6(r / s)^{3}+4(r / s)^{5}-(r / s)^{2}\right] \\
& +\frac{1}{512} K_{0}^{2} R \frac{s^{8}(0)}{s^{8}} \cdot s^{5} s^{2}\left[4(r / s)-10(r / s)^{3}+\frac{28}{3}(r / s)^{5}-3(r / s)^{7}\right] .
\end{aligned}
$$

Imposing the no-slip boundary condition results in the Reynolds equation for the pressure

$$
p_{s}^{\prime \prime}+4 \frac{s^{\prime}}{s} p_{s}^{\prime}=\frac{1}{96} K_{0}^{2} R \frac{s^{8}(0)}{s^{8}} \cdot s^{3} s^{\prime \prime}-\frac{1}{96} K_{0}^{2} R \frac{s^{8}(0)}{s^{8}} s^{2} s^{\prime}
$$

Integrating this, subject to $p_{s}^{(1)}(0)=0$, gives

$$
p_{s}^{\prime}=\frac{1}{96} K_{0}^{2} R\left[\frac{s^{8}(0)}{s^{8}} s s^{\prime}-\frac{s^{4}(0)}{s^{4}} s(0) s^{\prime}(0)\right] .
$$

To effect solution of (4.4) involves substitution of known quantities in the right-hand side of (4.4), taking the limit as $\sigma \rightarrow 0$ where necessary, and integrating once. The resulting equation can now be solved simultaneously with (4.4). 
Equation (4.5) can be tackled similarly. The results are

$$
\begin{aligned}
f_{s}^{(1)}=- & \frac{1}{3456} \alpha G \cos \chi P R K_{0} q_{2} \cdot \frac{s^{4}(0)}{s^{4}} \cdot s^{7} s^{\prime} \\
& \times\left[\frac{43}{80}-\frac{47}{40}(r / s)^{2}+\frac{3}{4}(r / s)^{4}-\frac{1}{8}(r / s)^{6}+\frac{1}{80}(r / s)^{8}\right]+O\left(\sigma^{1 / 2}\right) \\
g_{s}^{(1)}= & \frac{1}{3456} G \cos \chi P R K_{0} q_{2} \cdot \frac{s^{4}(0)}{s^{4}} \cdot s^{7} s^{\prime} \\
& \times\left[\frac{43}{80}-\frac{141}{40}(r / s)^{2}+\frac{15}{4}(r / s)^{4}-\frac{7}{8}(r / s)^{6}+\frac{9}{80}(r / s)^{8}\right]+O\left(\sigma^{1 / 2}\right)
\end{aligned}
$$

and

$$
\begin{aligned}
& f_{s}^{(2)}=\frac{1}{160.48^{2}} G \cos ^{2} \chi q_{2}^{2} s^{9}(\sigma / 2)^{1 / 2} \\
& \times\left[\frac{17}{96}(r / s)-\frac{3}{8}(r / s)^{3}+\frac{5}{24}(r / s)^{5}-\frac{1}{96}(r / s)^{9}+O(\sigma)\right] \\
& g_{s}^{(2)}=- \frac{1}{160.48^{2}} G \cos ^{2} \chi q_{2}^{2} s^{9}(\sigma / 2)^{1 / 2} \\
& \times\left[\frac{17}{96}(r / s)-\frac{3}{4}(r / s)^{3}+\frac{5}{8}(r / s)^{5}-\frac{5}{96}(r / s)^{9}+O(\sigma)\right] .
\end{aligned}
$$

In (4.12) we have left out terms of order $O\left(\sigma^{1 / 2}\right)$ as opposed to previous computations. These terms may be computed without many complexities. However, for the flow velocity we are primarily interested in the axial component, which convects the nutrients of the blood to various parts of the body in systemic circulation.

\section{Steady state streaming for large $\sigma$}

For reasons advanced above, we shall only consider the solutions to the temperature and axial velocity of equations (4.1) to (4.3) when $\sigma$ is large. Now it is no longer possible to construct uniformly-valid solutions, as a result of the presence of a boundary layer of thickness $(\sigma / 2)^{-1 / 2}$ and coordinate

$$
\eta=(\sigma / 2)^{1 / 2}(s-r)
$$


Thus, retaining small letters for the outer problem and adopting capital letters for the inner problem, equations (4.1) to (4.3) split into the two groups:

$$
\begin{gathered}
\frac{\partial^{2} \gamma_{s}^{(0)}}{\partial r^{2}}+\frac{1}{r} \frac{\partial \gamma_{s}^{(0)}}{\partial r}=0 \\
\frac{\partial^{2} \gamma_{s}^{(1)}}{\partial r^{2}}+\frac{1}{r} \frac{\partial \gamma_{s}^{(1)}}{\partial r}-\frac{1}{r^{2}} \gamma_{s}^{(1)}=0 \\
\frac{\partial^{2} h_{s}^{(0)}}{\partial r^{2}}+\frac{1}{r} \frac{\partial h_{s}^{(0)}}{\partial r}=p_{s}^{\prime} \\
\frac{\partial^{2} h_{s}^{(1)}}{\partial r^{2}}+\frac{1}{r} \frac{\partial h_{s}^{(1)}}{\partial r}-\frac{1}{r^{2}}=0 \\
\frac{1}{r} \frac{\partial}{\partial r}\left(r f_{s}^{(0)}\right)=-\frac{\partial h_{s}^{(0)}}{\partial z}
\end{gathered}
$$

and

$$
\begin{aligned}
& \frac{\partial^{2} \Gamma_{s}^{(0)}}{\partial \eta^{2}}+\frac{1}{\left[\eta-(\sigma / 2)^{1 / 2} s\right]} \frac{\partial \Gamma_{s}^{(0)}}{\partial \eta} \\
& =K_{0} q_{2} \alpha^{1 / 2} P^{1 / 2} R s^{\prime} \cdot \frac{1}{\sigma^{2}} \operatorname{Re}\left\{(1-i)\left[e^{-(1-i) P^{1 / 2} \eta}-e^{-\chi \eta}+O\left(\sigma^{-1 / 2}\right)\right]\right\} \\
& \frac{\partial^{2} \Gamma_{s}^{(1)}}{\partial \eta^{2}}+\frac{1}{\left[\eta-(\sigma / 2)^{1 / 2} s\right]} \frac{\partial \Gamma_{s}^{(1)}}{\partial \eta}-\frac{1}{\left[\eta-(\sigma / 2)^{1 / 2} s\right]^{2}} \Gamma_{s}^{(1)} \\
& =\frac{8 G \cos \chi q_{2}^{2}}{\alpha P(1-\alpha P) s} \cdot \frac{1}{\sigma^{3}} \operatorname{Re}\left\{( 1 - i ) \left[\left(1-\alpha^{1 / 2} P^{1 / 2}\right) e^{-(1-i) \alpha^{1 / 2} P^{1 / 2} \eta}\right.\right. \\
& \left.\left.-e^{-2 \alpha^{1 / 2} p^{1 / 2} \eta}+\alpha^{1 / 2} p^{1 / 2} e^{-\chi \eta}+O\left(\sigma^{-1 / 2}\right)\right]\right\} \\
& \frac{\partial^{2} H_{s}^{(0)}}{\partial \eta^{2}}+\frac{1}{\left[\eta-(\sigma / 2)^{1 / 2} s\right]} \frac{\partial H_{s}^{(0)}}{\partial \eta} \\
& =-K_{0}^{2} R \frac{s^{4}(0)}{s^{4}} s^{\prime} \frac{1}{\sigma^{2}} \operatorname{Re}\left\{(1-i)\left[e^{-(1-i) \eta}-e^{-2 \eta}+O\left(\sigma^{-1 / 2}\right)\right]\right\}+P_{s}^{\prime} \\
& \frac{\partial^{2} H_{s}^{(1)}}{\partial \eta^{2}}+\frac{1}{\left[\eta-(\sigma / 2)^{1 / 2} s\right]} \frac{\partial H_{s}^{(1)}}{\partial \eta}-\frac{1}{\left[\eta-(\sigma / 2)^{1 / 2} s\right]^{2}} H_{s}^{(1)} \\
& =-\frac{8 G \cos \chi R \alpha_{1} K_{0}}{\alpha^{3 / 2} P^{3 / 2}(1-\alpha P)} \cdot \frac{s^{2}(0)}{s^{2}} \cdot \frac{1}{s} \cdot \frac{1}{\sigma^{3}} \\
& \operatorname{Re}\left\{(1-i)\left[\left(1-\alpha^{1 / 2} P^{1 / 2}\right) e^{-(1-i) \eta}+\alpha^{1 / 2} P^{1 / 2} e^{-2 \eta}-e^{-\tilde{x} \eta}+O\left(\sigma^{-1 / 2}\right)\right]\right\} \\
& \frac{1}{\left[\eta-(\sigma / 2)^{1 / 2} s\right]} \frac{\partial}{\partial \eta}\left\{\left[\eta-(\sigma / 2)^{1 / 2} s\right] F_{s}^{(0)}\right\}=\left.(\sigma / 2)^{-1 / 2} \frac{\partial H_{s}^{(0)}}{\partial z}\right|_{r}
\end{aligned}
$$


where

$$
\chi=\left(1+\alpha^{1 / 2} P^{1 / 2}\right)+i\left(1-\alpha^{1 / 2} P^{1 / 2}\right)
$$

and

$$
P_{s}^{\prime}=\frac{2}{\sigma} p_{s}^{\prime}
$$

The boundary and end conditions are

$$
\Gamma_{s}^{(0,1)}(0, z)=0=H_{s}^{(0,1}(0, z)=F_{s}^{(0)}(0, z), \quad P_{s}^{\prime}(0)=0 .
$$

The solutions to the outer and inner problems can be effected by standard techniques. The outer solutions are straightforward and are given by

$$
\begin{aligned}
& \gamma_{s}^{(0)}=a_{s}^{(0)} \quad \gamma_{s}^{(1)}=a_{s}^{(1)} r \\
& h_{s}^{(0)}=\frac{1}{4} p_{s}^{\prime}\left(r^{2}+b_{s}^{(0)}\right), \quad h_{s}^{(1)}=b_{s}^{(1)} r, \quad f_{s}^{(0)}=-\frac{1}{16} p_{s}^{\prime \prime}\left(r^{3}+2 b_{s}^{(0)} r\right)-\frac{1}{8} p_{s}^{\prime} b_{s}^{(0) \prime} r
\end{aligned}
$$

where $a_{s}^{(0,1)}$ and $b^{(0,1)}$ are arbitrary functions of $z$ to be determined. For the inner problem, on defining the exponential integral

$$
E_{m}(z)=\int_{1}^{\infty} e^{-z t} t^{-n} d t
$$

the solution of equations (5.2) and (5.3) may be put in the form

$$
\begin{aligned}
\Gamma_{s}^{(0)}=K_{0} q_{2} \alpha^{1 / 2} P^{1 / 2} R s^{\prime} & \frac{1}{\sigma^{2}} \\
\times \operatorname{Re}\left\{( 1 - i ) \left[A_{s}^{(0)}-\frac{1}{2 i \alpha P}\right.\right. & \left(e^{-(1-i) \alpha^{1 / 2} P^{1 / 2} \eta}-e^{-(1-i)(\sigma \alpha P / 2)^{1 / 2} s}\right. \\
& \left.\times E_{1}\left((1-i) \alpha^{1 / 2} P^{1 / 2}\left[\eta-(\sigma / 2)^{1 / 2} s\right]\right)\right) \\
& \left.\left.-\frac{1}{\chi^{2}}\left(e^{-\chi \eta}-e^{-\chi(\sigma / 2)^{1 / 2} s} E_{1}\left(\chi\left[\eta-(\sigma / 2)^{1 / 2} s\right]\right)\right)\right]\right\},
\end{aligned}
$$




$$
\begin{aligned}
\Gamma_{s}^{(1)}= & \frac{8 G \cos \chi R q_{2}^{2}}{\alpha P(1-\alpha P)} \cdot \frac{1}{\sigma^{3}} \operatorname{Re}[(1-i) \\
& \times\left(A_{s}^{(1)}\left[\eta-(\sigma / 2)^{1 / 2} s\right]-\frac{\left(1-\alpha^{1 / 2} P^{1 / 2}\right)}{8 i \alpha^{3 / 2} P^{3 / 2}}\right. \\
& \times\left\{(1-i) \alpha^{1 / 2} P^{1 / 2}+\frac{1}{\left[\eta-(\sigma / 2)^{1 / 2} s\right]}\right\} e^{-(1-i) \alpha^{1 / 2} P^{1 / 2} \eta} \\
& +\frac{1}{8 \alpha^{3 / 2} P^{3 / 2}}\left\{2 \alpha^{1 / 2} P^{1 / 2}+\frac{1}{\left[\eta-(\sigma / 2)^{1 / 2} s\right]}\right\} e^{-\alpha^{1 / 2} P^{1 / 2} \eta} \\
& \left.\left.+\frac{\alpha^{1 / 2} P^{1 / 2}}{\chi^{3}}\left\{\chi+\frac{1}{\left[\eta-(\sigma / 2)^{1 / 2} s\right]}\right\} e^{-\chi \eta}\right)\right],
\end{aligned}
$$

$$
\begin{aligned}
& H_{s}^{(0)}=\frac{1}{4} p_{s}^{\prime}\left\{\left[\eta-(\sigma / 2)^{1 / 2} s\right]^{2}-\right.\left.\frac{\sigma}{2} s^{2}\right\} \\
&-K_{0}^{2} R \frac{s^{4}(0)}{s^{4}} s^{\prime} \cdot \frac{1}{\sigma^{2}} \operatorname{Re}\left\{( 1 - i ) \left[B_{s}^{(0)}-\frac{1}{2 i}\left(e^{-(1-i) \eta}\right.\right.\right. \\
& \quad-e^{-(1-i)(\sigma / 2)^{1 / 2} s} E_{1}\left\{(1-i)\left[\eta-(\sigma / 2)^{1 / 2} s\right]\right\} \\
&-\left.\left.\left.\frac{1}{4}\left(e^{-2 \eta}-e^{-(2 \sigma)^{1 / 2} s} E_{1}\left\{2\left[\eta-(\sigma / 2)^{1 / 2} s\right]\right\}\right)\right)\right]\right\}
\end{aligned}
$$

$$
\begin{aligned}
H_{s}^{(1)}=-\frac{8 G \cos \chi R q_{2} K_{0}}{\alpha^{3 / 2} P^{3 / 2}(1-\alpha P)} \cdot \frac{s^{2}(0)}{s^{2}} \cdot \frac{1}{s} \cdot \frac{1}{\sigma^{3}} \operatorname{Re}\left[( 1 - i ) \left(B_{s}^{(1)}\left[\eta-(\sigma / 2)^{1 / 2} s\right]\right.\right. \\
-\frac{\left(1-\alpha^{1 / 2} P^{1 / 2}\right)}{8 i}\left\{1-i+\frac{1}{\left[\eta-(\sigma / 2)^{1 / 2} s\right]}\right\} e^{-(1-i) \eta} \\
+\frac{\alpha^{1 / 2} P^{1 / 2}}{8}\left\{2+\frac{1}{\left[\eta-(\sigma / 2)^{1 / 2} s\right]}\right\} e^{-2 \eta} \\
\left.\left.-\frac{1}{\bar{\chi}^{3}}\left\{\bar{\chi}+\frac{1}{\left[\eta-(\sigma / 2)^{1 / 2} s\right]}\right\} e^{-\bar{\chi} \eta}\right)\right] .
\end{aligned}
$$

$A_{s}^{(0,1)}$ and $B_{s}^{(0,1)}$ are functions of $z$ whose values follow from the homogeneous boundary conditions (5.6). 
To obtain the value for $P_{s}^{\prime}$, we now substitute equation (5.11) into equation (5.4). After integration, the result is

$$
\begin{aligned}
& F_{s}^{(0)}=\frac{1}{16(\sigma / 2)^{1 / 2}} P_{s}^{\prime \prime}\left\{\left[\eta-(\sigma / 2)^{1 / 2} s\right]^{3}-2(\sigma / 2) s^{2}\left[\eta-(\sigma / 2)^{1 / 2} s\right]\right\} \\
& -\frac{1}{4}(\sigma / 2)^{1 / 2} s s^{\prime} P_{s}^{\prime}\left[\eta-(\sigma / 2)^{1 / 2} s\right] \\
& +\frac{K_{0}^{2} R s^{4}(0)}{s^{4}} s^{\prime} \cdot \frac{1}{\sigma^{2}\left[\eta-(\sigma / 2)^{1 / 2} s\right]} \\
& \times \operatorname{Re}\left\{( 1 - i ) \left[\frac{1}{2 i}\left(\left[\eta-(\sigma / 2)^{1 / 2} s\right]+\frac{1}{1-i}\right) e^{-(1-i) \eta}\right.\right. \\
& +\frac{1}{4}\left(\left[\eta-(\sigma / 2)^{1 / 2} s\right]+\frac{1}{2}\right) e^{-2 \eta} \\
& -\frac{1-i}{2 i} e^{-(1-i)(\sigma / 2)^{1 / 2} s}\left(\frac{\left[\eta-(\sigma / 2)^{1 / 2} s\right]}{1-i} E_{2}\left\{(1-i)\left[\eta-(\sigma / 2)^{1 / 2} s\right]\right\}\right. \\
& +\frac{1}{(1-i)^{2}} E_{3}\left\{(1-i)\left[\eta-(\sigma / 2)^{1 / 2} s\right]\right\} \\
& \left.\left.\frac{1}{2}\left[\eta-(\sigma / 2)^{1 / 2} s\right]^{2} E_{1}[1-i)(\sigma / 2)^{1 / 2} s\right]\right) \\
& -\frac{1}{4} e^{-(2 \sigma)^{1 / 2} s}\left(\frac{\left\lfloor\eta-(\sigma / 2)^{1 / 2} s\right]}{2} E_{2}\left\{2\left[\eta-(\sigma / 2)^{1 / 2} s\right]\right\}\right. \\
& \left.\left.+\frac{1}{4} E_{3}\left\{2\left[\eta-(\sigma / 2)^{1 / 2} s\right]\right\}+\frac{1}{2}\left[\eta-(\sigma / 2)^{1 / 2} s\right]^{2} E_{1}\left[-(2 \sigma)^{1 / 2}\right]\right)\right] \\
& \left.+O\left(\sigma^{-1 / 2}\right)\right\} \text {. }
\end{aligned}
$$

Imposing boundary condition (5.6) on (5.12) leads to the Reynolds equation

$$
\begin{aligned}
P_{s}^{\prime \prime}+4 \frac{s^{\prime}}{s} P_{s}^{\prime}= & 4 K_{0}^{2} R \frac{s^{4}(0)}{s^{4}} \cdot \frac{s^{\prime}}{s^{4}} \cdot \frac{1}{(\sigma / 2)^{7 / 2}} \\
& \cdot \operatorname{Re}\left\{( 1 - i ) \left[\frac{1}{2 i}\left(-(\sigma / 2)^{1 / 2} s+\frac{1}{1-i}\right)+\frac{1}{4}\left(-(\sigma / 2)^{1 / 2} s+\frac{1}{2}\right)\right.\right. \\
& -\frac{1-i}{2 i} e^{-(1-i)(\sigma / 2)^{1 / 2} s\left(\frac{-(\sigma / 2)^{1 / 2} s}{1-i} E_{2}\left[-(1-i)(\sigma / 2)^{1 / 2} s\right]\right.} \\
& +\frac{1}{(1-i)^{2}} E_{3}\left[-(1-i)(\sigma / 2)^{1 / 2} s\right] \\
& \left.+\frac{1}{2}(\sigma / 2) s^{2} E_{1}\left[-(1-i)(\sigma / 2)^{1 / 2} s\right]\right) \\
& -\frac{1}{4} e^{-(2 \sigma)^{1 / 2} s}\left(\frac{-(\sigma / 2)^{1 / 2} s}{2} E_{2}\left[-(2 \sigma)^{1 / 2} s\right]+\frac{1}{4} E_{3}\left[-(2 \sigma)^{1 / 2} s\right]\right. \\
& \left.\left.\left.+\frac{1}{2}(\sigma / 2) s^{2} E_{1}\left[-(2 \sigma)^{1 / 2} s\right]\right)\right]\right\} .
\end{aligned}
$$


Integrating this equation subject to the end condition (5.6), we have

$$
\begin{aligned}
P_{s}^{\prime}= & 4 K_{0}^{2} R \frac{s^{4}(0)}{s^{4}} \cdot \frac{1}{(\sigma / 2)^{7 / 2}} \\
& +\operatorname{Re}\left[( 1 - i ) \left(\frac{1}{2 i}\left[\frac{1}{2}(\sigma / 2)^{1 / 2}\left(\frac{1}{s^{2}}-\frac{1}{s^{2}(0)}\right)-\frac{1}{3(1-i)}\left(\frac{1}{s^{3}}-\frac{1}{s^{3}(0)}\right)\right]\right.\right. \\
& +\frac{1}{4}\left[\frac{1}{2}(\sigma / 2)^{1 / 2}\left(\frac{1}{s^{2}}-\frac{1}{s^{2}(0)}\right)-\frac{1}{6}\left(\frac{1}{s^{3}}-\frac{1}{s^{3}(0)}\right)\right] \\
& -\frac{1-i}{2 i}\left\{\frac{-(\sigma / 2)^{1 / 2}}{1-i} e_{2}\left[-(1-i)(\sigma / 2)^{1 / 2}\right]\right. \\
& \left.+\frac{1}{(1-i)^{2}} e_{3}\left[-(1-i)(\sigma / 2)^{1 / 2}\right]+\frac{1}{2}(\sigma / 2) e_{1}\left[-(1-i)(\sigma / 2)^{1 / 2}\right]\right\} \\
& -\frac{1}{4}\left\{-\frac{1}{2}(\sigma / 2)^{1 / 2} e_{2}\left[-(2 \sigma)^{1 / 2}\right.\right. \\
& \left.\left.\left.+\frac{1}{4} e_{3}\left[-(2 \sigma)^{1 / 2}\right]+\frac{1}{2}(\sigma / 2) e_{1}\left[-(2 \sigma)^{1 / 2}\right]\right\}\right)\right],
\end{aligned}
$$

where

$$
\begin{aligned}
e_{n}(-\alpha) & =\int_{s(0)}^{s} e^{-\alpha u} \frac{E_{n}(-\alpha u)}{u^{n+1}} d u \\
& \sim \frac{1}{n+1}\left(\frac{1}{s^{n+1}}-\frac{1}{s^{n+1}(0)}\right) \cdot \frac{1}{\alpha} \quad \text { for large } \alpha .
\end{aligned}
$$

Finally, matching the outer and inner solutions, we find that

$$
\begin{aligned}
& a_{s}^{(0)}=K_{0} q_{2} \alpha^{1 / 2} P^{1 / 2} R s^{\prime} \frac{1}{\sigma^{2}} \operatorname{Re}\left[(1-i) A_{s}^{(0)}\right] \\
& a_{s}^{(1)}=-\frac{G \cos \chi R q_{2}^{2}}{\alpha P(1-\alpha P)} \cdot \frac{1}{(\sigma / 2)} \operatorname{Re}\left[(1-i) A_{s}^{(1)}\right] \\
& b_{s}^{(0)}=-s^{2}, \quad b_{s}^{(1)}=\frac{G \cos \chi R q_{2} K_{0}}{\alpha^{3 / 2} P^{3 / 2}(1-\alpha P)} \cdot \frac{s^{2}(0)}{s^{2}} \cdot \frac{1}{s} \cdot \frac{1}{(\sigma / 2)^{5 / 2}} \operatorname{Re}\left[(1-i) B_{s}^{(1)}\right] .
\end{aligned}
$$

The solutions are now complete, since $p_{s}^{\prime}$ could be obtained from equation (5.5) and (5.13) by discarding terms which decay exponentially in equation (5.13).

\section{Discussion}

The modification to the mean flow, as a result of axial temperature variation of the tube wall, has been noted. Quantitatively the discussion of the problem for the cases:
(i) $f(z)=e^{ \pm \gamma z}$,
(ii) $f(z)= \pm \gamma z, \gamma>0$; 
is not very different from that presented by Bestman [1] when the wall temperature is uniform. Therefore the present discussion will be limited to the steady streaming flow for which the wall temperature variation has no effect.

The problem of free convection heat transfer has far-reaching effects on the flow in a tube of varying section. Indeed, the boundary condition for the velocity at the wall, in non-dimensional form, as discussed by Manton [4], should be

$$
w+\varepsilon^{2} s^{\prime}=u=0, \quad v=0 \quad r=s(z) .
$$

This is also the form adopted by Bestman [2] in the analysis of oscillatory flow in a tube of varying section in absence of heat transfer. In this case the basic perturbed pressure gradient corresponded with the forcing pressure gradient. All higher pressure gradients are zero.

In the presence of heat transfer, which is the primary concern of this paper, it is necessary to take $u$ and $w$ as zero at the tube wall. The perturbed pressure gradients are now deduced by lubrication theory, so that even the basic order pressure gradients are different from zero. If the amplitude of the oscillatory forcing pressure gradient transcends over the mean pressure gradient (that is $K_{0} \gg C_{0}$ ) then the consequence of this at a local constriction (stenosis), such that $s^{\prime}=0$ at the constriction, is to offset the trapping effect and consequent thrombus formation discussed by Bestman [2].

A cursory excursion into the literature reveals the following characteristics of blood in man:

\begin{tabular}{ll}
\hline \multicolumn{1}{c}{ Characteristics of blood } & numerical value \\
\hline characteristic velocity in aorta & $40 \mathrm{~cm} / \mathrm{sec}$ \\
kinematic viscosity $(\nu)$ & $0.04 \mathrm{~cm}^{2} / \mathrm{sec}$ \\
typical aorta radius & $0.04 \mathrm{~cm}$ \\
Prandtl number $(P)$ & 25 \\
\hline
\end{tabular}

For simplicity we take $\varepsilon=0.01$ and $\beta=1 / T_{\infty}$ where $T_{\infty}$ is taken as $38^{\circ} \mathrm{C}$. Thus for a pleasant $20^{\circ} \mathrm{C}$, the Grashof number $G=5.3 \ldots$ while for a hot $40^{\circ} \mathrm{C}$ it is $0.6 \ldots$

We shall consider numerical discussion for a locally constricted tube of the form

$$
s(z)=1-(1 / 2) \exp \left(-z^{2}\right) .
$$

We take $K_{0}=1, \phi=0$ and with the origin $(z=0)$ at the constriction, the value of $s(0)$ (at the inlet assumed far away from the constriction) may be taken as unity. The other parameters of the problem are varied to simulate different physical situations. We shall limit our discussions to the case of small $\sigma$. When $\sigma$ is large, the situation remains very similar except that the temperature and 


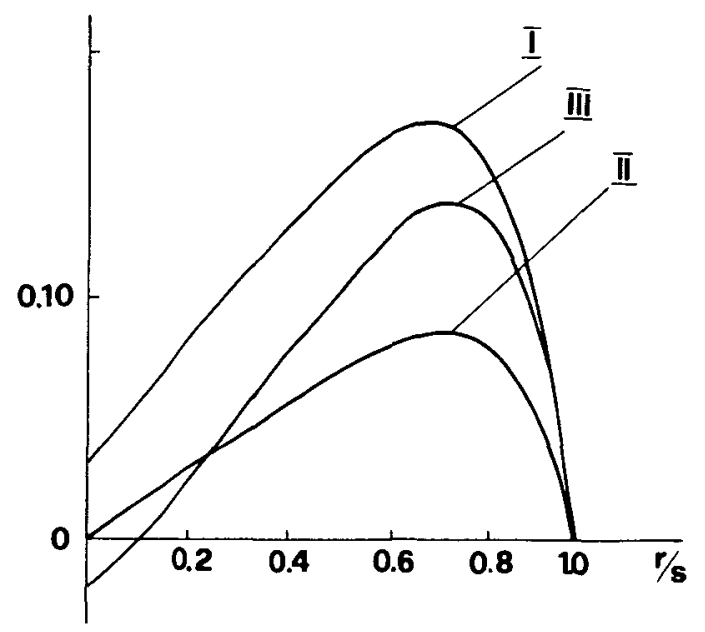

FIGURE 1. Steady streaming axial velocity profile

$$
\begin{aligned}
& R=40, G=5, \alpha_{1}=1.0, P=25, \sigma=0.1 \\
& z \\
&-0.5 \text { I } \\
& 0.0 \text { II } \\
& 0.5 \text { III }
\end{aligned}
$$

velocities involved are much smaller as evidenced by the analysis in Sections 3 and 4.

In Figure 1, the steady streaming axial velocity is plotted across the tube upstream of the constriction, at the constriction, and downstream of the construction, with $P=25$ and $R=40$, as may correspond to blood. If $P=7$ and $R=10$, which may correspond to water, the various flow variables reduce by approximately $5 \%$. This reduction is quite small when compared to the product $\varepsilon P R$, which takes the value 0.7 for water and 10 for blood. Apart from a small backflow near the centre of the tube downstream of the constriction, all fluid flows in the desired direction. This could be contrasted with the case of zero heat transfer (see [4]) in which the fluid flows in the right direction downstream of the constriction, while upstream of the constriction it flows in the reverse direction, the flow velocity being zero at the constriction. This is the phenomenon of trapping, with possible thrombus formation at the constriction. Free convection heat transfer annihilates this thrombus formation, provided the mean flow is small in comparison with the oscillatory flow, $K_{0} \gg C_{0}$.

The steady streaming velocity distribution for various values of the parameter are depicted in Figure 2. Increase in the Reynolds number causes a corresponding increase in velocity, while increase in the Prandtl number has negligible effect 


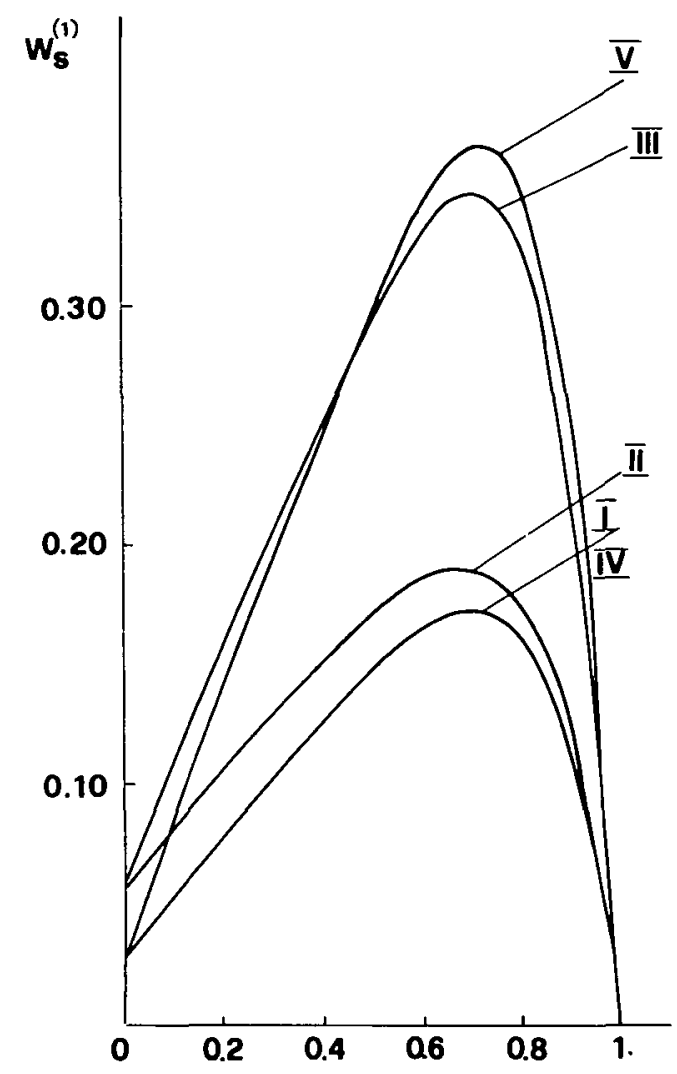

FIGURE 2. Steady streaming axial velocity profile

$$
\begin{array}{rrrrr}
\multicolumn{5}{c}{z=-0.5, q_{2}=1.0} \\
R & G & P & \sigma & \\
40 & .5 & 25 & 0.1 & \text { I } \\
60 & .5 & 25 & 0.1 & \text { II } \\
60 & 1.0 & 25 & 0.1 & \text { III } \\
40 & .5 & 25 & 0.1 & \text { IV } \\
40 & .5 & 40 & 0.5 & \text { V }
\end{array}
$$

on the velocity. Also, increase in the free convection parameter $(G)$ and $\sigma$ cause a more drastic increase in the velocity.

It was also observed that the temperature at $z=-0.5$ is negative, whereas it is positive (but virtually equal in magnitude) when $z=0.5$. At the throat the temperature is zero. When $R$ and $P$ increase, the temperature increases while increase in $G$ and $\sigma$ has negligible effect on the temperature. When the source and sink interchange their role, for example $G=-5, q_{2}=-1$, the velocity is 
unaltered while the temperature distribution is reflected symmetrically about the $r / s$-axis.

It is a sobering thought that for several years physicians have used a hot water bottle in areas of suspected thrombus formation. The quantitative reason has been revealed by this analysis.

Finally we compare the steady streaming solution of Section 4 with the basic approximation arising from the mean pressure as given in (2.14). While the temperature and axial velocity in (2.14) are free from any roll, the buoyancy contribution in the steady streaming temperature and axial velocity gives rise to a double longitudinal roll system with the centre line as the axis of the roll. However this double roll system manifests itself in the radial and azimuthal velocity components of the mean forcing pressure. For the steady streaming radial and azimuthal velocity components, the free convection contribution is in two parts; a double roll system and four separate roll systems, one in each quadrant in the vertical plane.

\section{Acknowledgement}

This work was supported financially by the Federal University of Technology, Bauchi under the vote $303 / 4 / 3$. The author is grateful to physiologist Syder Nnodim for helpful discussions he had with him while on the academic staff of Unitech., Port Harcourt. Dr. Victor Adelakoun of Fedunitech Bauchi also provided simulating discussions, while the referees' comments lead to a considerable improvement on an earlier draft.

\section{Appendix}

$$
\begin{aligned}
& \text { Addition term }+\frac{256}{C^{(0) 2} s^{8}(0)} \\
& \begin{array}{r}
G \sin \chi\left\{\lambda f_{z} s^{3}\left[\frac{1}{2}(r / s)-\frac{1}{4}(r / s)^{3}\right]\right. \\
{[1+\lambda f(z)] s^{2} s_{z}(r / s)} \\
\left.+\frac{1}{32} q_{1}^{2} s^{3} s_{z}\left[\frac{9}{2}(r / s)-(r / s)^{2}-\frac{1}{6}(r / s)^{5}\right]\right\}
\end{array} \\
& \begin{array}{r}
G_{1}^{(1)}=-\frac{1}{25200} P G \cos \chi \lambda s^{3} f_{z z}\left[16(r / s)^{2}-35(r / s)^{4}+35(r / s)^{6}-16(r / s)^{8}\right] \\
-\frac{1}{5040} P G \cos \chi \lambda s^{2} f_{z}\left[35(r / s)^{4}-35(r / s)^{6}+16(r / s)^{8}\right]-\frac{\partial}{\partial r}\left(r F_{1}^{(1)}\right),
\end{array}
\end{aligned}
$$




$$
\begin{aligned}
& F_{1}^{(1)}=\frac{1}{254200} P G \cos \chi \lambda s^{3} f_{z z}\left\{16\left[1-(r / s)^{2}\right]-\frac{1}{24}\left[105+70(r / s)^{2}-175(r / s)^{4}\right]\right. \\
& \left.+\frac{1}{48}\left[140+105(r / s)^{2}-245(r / s)^{6}\right]-\frac{1}{5}\left[1+8(r / s)^{2}-9(r / s)^{8}\right]\right\} \\
& +\frac{1}{5040} P G \cos \chi \lambda s^{2} f_{z}\left\{\frac{1}{24}\left[105+70(r / s)^{2}-175(r / s)^{4}\right]\right. \\
& \left.-\frac{1}{48}\left[150+105(r / s)^{2}-245(r / s)^{6}\right]+\frac{1}{5}\left[1+8(r / s)^{2}-9(r / s)^{8}\right]\right\} \\
& -\frac{1}{360} R G \cos \chi \lambda C_{0} s^{4}(0) s^{2} s_{z} f_{z} \\
& \times\left\{\frac{7}{16}(r / s)^{2} \ln (r / s)+\frac{1}{32}\left[1+5(r / s)^{2}-6(r / s)^{4}\right]\right. \\
& -\frac{1}{1050}\left[291-485(r / s)^{2}+94(r / s)^{5}\right]-\frac{1}{128}\left[6-9(r / s)^{2}+3(r / s)^{6}\right] \\
& \left.+\frac{1}{1470}\left[205-287(r / s)^{2}+82(r / s)^{7}\right]\right\} \\
& +\frac{2}{45} R G \cos \chi \lambda s^{3} s_{z} f_{z z}\left\{\frac{1}{8}(r / s)^{2} \ln (r / s)+\frac{1}{16}\left[1-(r / s)^{2}\right]\right. \\
& -\frac{1}{96}\left[17-34(r / s)^{2}+17(r / s)^{4}\right]+\frac{1}{525}\left[51-85(r / s)^{2}+34(r / s)^{4}\right] \\
& \left.+\frac{1}{24}\left[2-3(r / s)^{2}+(r / s)^{2}+(r / s)^{6}\right]-\frac{1}{441}\left[25-35(r / s)^{2}+10(r / s)^{7}\right]\right\} \\
& +\frac{2}{45} R G \cos \chi \lambda s^{2} s_{z} f_{z}\left\{\frac{1}{16}(r / s)^{2} \ln (r / s)+\frac{1}{8}\left[1-(r / s)^{2}\right]\right. \\
& -\frac{1}{32}\left[15-30(r / s)^{2}+15(r / s)^{4}\right] \\
& \left.+\frac{1}{128}\left[10-15(r / s)^{2}+5(r / s)^{6}\right]-\frac{1}{735}\left[40-56(r / s)^{2}+16(r / s)^{7}\right]\right\} \\
& G_{2}^{(1)}=-\frac{1}{2} r \frac{\partial}{\partial r}\left(r F_{2}^{(1)}\right)
\end{aligned}
$$




$$
\begin{aligned}
& F_{2}^{(1)}=-\frac{1}{1120} P G \cos \chi \lambda C_{0} s^{4}(0) f_{z}\left[\frac{1}{2}(r / s)-\frac{3}{2}(r / s)^{3}+(r / s)^{4}\right] \\
&+\frac{1}{252.96 .10^{3}} P G \cos \chi q_{1} \lambda s^{7} f_{z}[79(r / s) \\
&-\frac{1}{69120} R G \cos ^{2} \chi \lambda q_{1} s^{8} f_{z}\left\{\frac{5}{8}(r / s)^{3} \ln (r / s)+\frac{5}{16}\left[r / s-(r / s)^{3}\right]\right. \\
&+ \frac{23}{210}\left[r / s-3(r / s)^{3}+2(r / s)^{4}\right]+\frac{1}{315}\left[138(r / s)-230(r / s)^{3}+92(r / s)\right] \\
&-\frac{13}{192}\left[r / s-2(r / s)^{3}+(r / s)^{5}\right]-\frac{211}{960}\left[2(r / s)-3(r / s)^{3}+(r / s)^{7}\right] \\
&+\left.-\frac{1}{3465}\left[305(r / s)-427(r / s)^{3}+122(r / s)^{8}\right]\right\} \\
&+\frac{1}{3100} R G \cos ^{2} \chi \lambda^{2} s^{7} f_{z}^{2}\left\{\frac { 1 1 } { 1 9 2 } \left[(r / s)-2(r / s)^{3}+(r / s)\right.\right. \\
&\left.+62(r / s)-63(r / s)^{3}+21(r / s)^{7}\right]-\frac{1}{14}\left[(r / s)-3(r / s)^{3}+2(r / s)^{4}\right] \\
&\left.-\frac{287}{1890}\left[3(r / s)-5(r / s)^{3}+2(r / s)^{6}\right]\right\}
\end{aligned}
$$

\section{References}

[1] A. R. Bestman, "Low Reynolds number flow in a heated tube of varying section", $J$. A ustral. Math. Soc. (Ser. B), 25 (1983) 244-260.

[2] A. R. Bestman, "Oscillatory non-Newtonian flow in tubes of slowly varying section", Acta Mechanica, 45 (1982) 17-30.

[3] P. Hall, "Unsteady viscous flow in a pipe of slowly varying cross-section", J. Fluid Mech., 67 (1974) 209-226.

[4] M. J. Manton, "Low Reynolds number flow in slowly varying axisymmetric tubes", $J$. Fluid Mech., 49 (1971) 451-459. 